\title{
A graphical exploration of the general performance indices for bending stiffness design
}

\author{
Damiano Pasini \\ McGill University \\ Department of Mechanical Engineering \\ Montreal, Quebec, H3A 2K6, Canada \\ tel: 514-398-6295 email: damiano.pasini@mcgill.ca
}

\section{Abstract}

$\frac{E}{\rho}, \frac{E^{1 / 3}}{\rho}$, and $\frac{E^{1 / 2}}{\rho}$ are not the only material indices to optimize the mass of a stiff beam. The general index to select the material for any arbitrary scaling of a cross-section is $\frac{E^{q}}{\rho}$, where $q$ is a function of the cross-section size. The result has been recently obtained by using the method of Shape Transformers, which measure the area, $\psi_{A}$, and the second moment of area, $\psi_{I}$, of a cross-section regardless of size. Their definition permits the general indices $\frac{\psi_{I}{ }^{q}}{\psi_{A}}$ for shape selection and $\frac{\left(E \psi_{I}\right)^{q}}{\rho \psi_{A}}$ for material and shape co-selection for any cross-section scaling.

This paper examines the use of the indices $\frac{E^{q}}{\rho}$ and $\frac{\psi_{I}^{q}}{\psi_{A}}$ for graphical selection. The effect of scaling is explored on property charts $E$ vs. $\rho$ and $\psi_{I}$ vs. $\psi_{A}$. As an alternative, limiting regime maps are proposed to illustrate how the performance ranking of materials and shapes varies in the design space with the relative change of the cross-section sizes. The maps facilitate selection for any scaling condition.

\section{Introduction}

Scaling the size of a cross-section affects the selection of the lightest material and shape for a stiff beam. The index of material selection, for example, changes its expressions with certain assumptions regarding the cross-section scaling. $\frac{E}{\rho}$ is to be maximised when the beam cross-section has a prescribed height and free width; the index turns out to be $\frac{E^{1 / 3}}{\rho}$ if the width is given and its depth can be scaled vertically; while constraining the ratio of the cross-section sizes yields $\frac{E^{1 / 2}}{\rho}$.

Performance indices are often combined with design charts to visualize how performance changes with design variables [1-3]. In material selection, Ashby [4-5] has shown that material indices can be developed in many applications to optimize the performance of a component and can be plotted on material charts to guide selection. For instance, in least weight stiffness design plotting the above indices on a chart $E$ vs $\rho$ provides insight into how material performance changes with the three crosssection scaling.

More recently, a methodology of Shape Transformers has been introduced for material and shape selection [6]. The approach can be applied to obtain performance indices for cross-sections subjected to any scaling transformation. For example, in bending stiffness design $\frac{E^{q}}{\rho}$ and $\frac{\psi_{I}^{q}}{\psi_{A}}$ are general 
indices for material and shape selection, while $\frac{\left(E \psi_{I}\right)^{q}}{\rho \psi_{A}}$ permits their co-selection. $q$ is a parameter governing the scaling transformation including the one that is neither horizontal, nor vertical, nor proportional, but arbitrary.

This paper examines the visualization of the general indices for material and for shape selection. It begins by describing the reasons for imposing a certain scaling and clarifying the implications. Next, a summary of the fundamentals of the Shape Transformers method is presented and general indices for any cross-section scaling are given. The parts following focus on selection charts. The issues of scaling are investigated on material property charts and Shape Transformer charts. Advantages and limitations of such charts, as well as alternative maps of limiting regimes, are presented. The latter illustrate how the ranking of materials and shapes throughout the whole design space varies with the relative changes of the cross-section size. The maps gives insight into how scaling impacts material and shape performance.

\section{The impact of scaling on the selection indices}

Figure 1 shows examples of scaled crosssections. Those on the left-hand side are all rectangles with different materials, while the shape varies for those on the right. Since the cross-sections also differ according to size, it can be observed that there is a relative cross-section scaling. $\mathrm{S}$ and $\mathrm{A}$, for example, represent Steel and Aluminium crosssections with sizes that are proportionally scaled. S and $\mathrm{G}$ (GFRP) are scaled in a vertical direction, while the rectangle, $\mathrm{R}$, and the ellipse, $\mathrm{E}$, are constrained to a horizontal scaling. When the relative scaling is in neither one of the above three directions, then it is arbitrary. $\mathrm{S}$ and $\mathrm{Z}$, as well as $\mathrm{R}$ and $\mathrm{D}$ are two examples.

There can be a variety of reasons for imposing a certain scaling to a cross-section [6-9]. The design may require specific geometric constraints to be satisfied, as well as size and shape availability and type of applied loading must be considered. For example, the design of a floor or of a wall structure may impose height or width constraints that restrict the cross-section scaling to a vertical or horizontal direction. Another case is that of a beam subjected to bending along both axes of its equiaxed crosssection. The design may be optimised by restraining the height to depth ratio. Besides, arbitrary scaling is not unusual in the design of tightly constrained structures because restrictions can be applied even at an inclined angle to the horizontal $[7,8]$.

A variety of performance indices have been proposed to rank material and shape efficiency of cross-sections [4-10]. The indices are combinations of design variables and are obtained for a given set of requirements and under certain assumptions. Among these, scaling plays a major role because it affects the index expression used for optimum selection. An example of this is the change of the power in the index used to optimise a beam with given stiffness. The index exponent, $q$, changes value with the relative scaling of the cross-sections, as illustrated in Figure 1. The vertical scaling between cross-sections $\mathrm{S}$ and $\mathrm{G}$ results in $q_{v}=1 / 3$, the proportional scaling of $\mathrm{S}$ and A causes $q_{p}$ to be $1 / 2$, and for horizontally scaled cross-sections, $q_{h}=1 . q$ is an arbitrary value for any other scaling, such as for directions $\mathrm{S}-\mathrm{Z}$ and R-D in Figure 1.

To determine the general expression of the scaling parameter $q$ for any scaling condition, we need a method. This is presented in the following section.

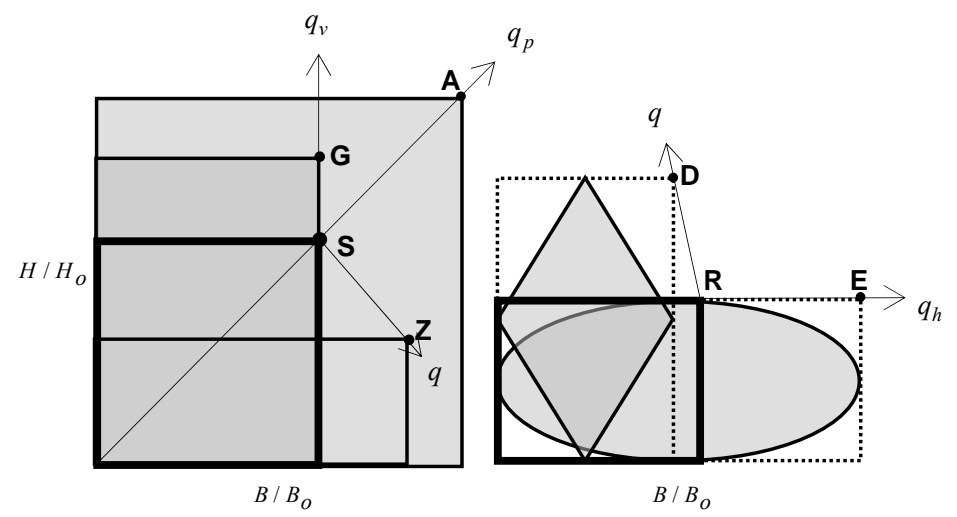

Figure 1. The power $q$ of the selection indices is governed by the relative scaling of cross-sections.

\subsection{The method of Shape Transformers}

Shape Transformers are dimensionless measures of the geometric quantities of a cross-section. They have been introduced to specify shape properties that do not change with any scaling transformation $[6$, $10]$.

The method is based on the idea that a crosssection can be described by two separate entities. The first one describes the size of a cross-section and is described by a rectangle whose size is given by the height and width of the cross-section. This rectangle, called the Envelope, is labelled $D$ in Figure 2, and is the entity related to the scaling. The second entity characterizes the shape properties, $S$, of the figure enclosed in the Envelope. These properties are called 
Shape Transformers; they measure the geometric quantities of a cross-section regardless of size and thus are invariant to scaling.

Figure 2 shows the Envelope and the Shape of three cross-sections. They all have different Shape, $S$. $D$ for cross-section (c) differs from that of crosssections (a) and (b), while there is no relative scaling between (a) and (b) because $D$ is the same.

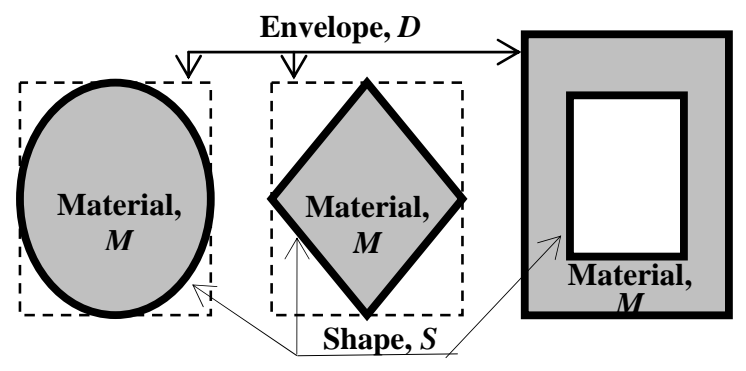

(a)

(b)

(c)

Figure 2. The material, $M$, the Shape, $S$, and the Envelope, $D$, of cross-sections.

\subsection{Shape Transformers definition}

A Shape Transformer characterizes a geometric quantity of a cross-section, $G$, regardless of its size, $D$. If the geometric quantity of the Envelope is $G_{D}$, then a Shape Transformer is defined as:

$$
S=\frac{G}{G_{D}}
$$

When $G$, for example, represents the area, $A$, and the second moment of area about the bending axis, $I$, of a cross-section, the Shape Transformers for the area, $\psi_{A}$, and for the second moment of area, $\psi_{I}$, by equation (1) are:

$$
\left\{\begin{array}{l}
\psi_{A}=\frac{A}{A_{D}} \\
\psi_{I}=\frac{I}{I_{D}}
\end{array}\right.
$$

Shape Transformers have been presented for a variety of cross-sections $[6,9,10]$. Their expressions are reported in Table 1 for some cross-sectional shapes where the Envelope, $D$, is described by the cross-section sizes, $B$ and $H$.

Shape Transformers define classes of shapes in a way that is similar to the way in which materials are classified. Within a class, there are the basic solid Shape and its derived hollow ones. These are obtained by removing a material layer with Shape $\mathrm{S}$ that is the same of its basic solid one. Each class has a domain of the Shape Transformers. The limits of a domain are zero, i.e. empty Envelope, and the $S$ value of the solid Shape (Table 1).

For any rectangle, the Shape completely fills the Envelope, $G=G_{D}$, and $S$ is unity such that:

$$
\left\{\begin{array}{l}
A=A_{D} \rightarrow \psi_{A}=1 \\
I=I_{D} \rightarrow \psi_{I}=1
\end{array}\right.
$$

For any other shape, $S$ is less than unity. Solid cross-sections have constant values of $S$, while $S$ for a hollow section changes value with the portion of material layer subtracted from its solid shape.

Shape Transformers have been used to rank the performance of cross-sections with the same Envelope. An Envelope Efficiency parameter, that assesses how efficiently a material is placed within an Envelope, has been introduced [6,9] as:

$$
\lambda=\frac{\psi_{A}}{\psi_{I}}
$$

Table 1 reports ranges for expression (2b). It is noted that these are theoretical because they do not consider manufacturing constraints and material efficiency limits. The graphical connotation of $\lambda$ and its limits have been examined for each class of Shape and visualized on charts $\psi_{A}$ vs. $\psi_{I}[9,10]$.

\subsection{Envelope multiplicators}

Scaling between cross-sections can be assessed by the relative changes of the Envelope sizes. We chose the rectangle as the reference cross-section from which any cross-section can be scaled. The reasons for this choice are that a rectangle is also the Envelope of the Shape, and the Shape Transformers of a rectangle are unity.

The scaling of a generic cross-section with Envelope sizes $B$ and $H$ relative to the reference with sizes $B_{o}$ and $H_{o}$, is described by the multiplicators, $u$ and $v$, as defined below:

$$
\left\{\begin{array}{l}
u=\frac{B}{B_{o}}=\frac{b}{b_{o}} \\
v=\frac{H}{H_{o}}=\frac{h}{h_{o}}
\end{array}\right.
$$

where $b$ and $b_{o}, h$ and $h_{o}$ are the dimensions of the internal layer for hollow or open cross-sections (Table 1). 
Table 1. Shape Transformers for the area and second moment of area of cross-sections with $c=b / B$ and $d=h / H$

\begin{tabular}{c|c|c|c|c|c} 
Cross-section & $\psi_{A}=A / A_{D}$ & $\psi_{A}$ range & $\psi_{I}=I / I_{D}$ & $\psi_{I}$ range & $\begin{array}{c}\lambda=\psi_{I} / \psi_{A} \\
\text { range }\end{array}$ \\
\hline & 1 & No range & No range & No \\
range
\end{tabular}

\subsection{The fundamental rationale}

The definition of Shape Transformers allows factors related to scaling to be separated from those related to Shape. The uncoupling can be advantageous when writing the Equation of Mechanics because each variable may be individually optimised.

In general, an Equation of Mechanics, E.M., can often be expressed in terms of material properties, $M$, geometry, $G$, and problem specifications, $F$, in the form:

$$
\text { E.M. }=F \times M \times G
$$

If the Shape Transformer definition (1) is rearranged and used to substitute $G$ in equation (4), then the E.M. can be written as:

$$
\text { E.M. }=F \times M \times S \times G_{D}
$$

where $F$ are the design specifications; $M$ are the material properties, such as the density, $\rho$, and the Young's Modulus, E; $S$ are the Shape Transformers of the cross-section, such as $\psi_{A}$ and $\psi_{I}$; and $G_{D}$ are the geometric quantities of the Envelope, $D$.

The principle of the Shape Transformers methodology is expressed by equation (5). $F, M, S$, and $D$ can be often uncoupled in an Equation of Mechanics [8], and thus the role that each design variable has regarding performance can be readily examined.

\section{Selection indices for scaled cross- sections}

This section presents general expressions of performance indices for material and shape selection in bending stiffness design. The indices are general and can be applied to design a beam subjected to any scaling of its cross-section.

Table 2. Density, $\rho$, and Young's Modulus, $E$.

\begin{tabular}{|ccc|}
\hline Material & $\rho\left(\mathrm{Mg} / \mathrm{m}^{3}\right)$ & $E(\mathrm{GPa})$ \\
\hline STEEL & 7.9 & 210 \\
\hline ALUMINIUM & 2.71 & 70 \\
\hline GFRP & 1.8 & 30 \\
\hline
\end{tabular}




\subsection{Material selection}

We consider the selection of the best material for a light and stiff beam of a given length, and arbitrarily chose two generic cross-sections with the same Shape, such as A and Z in Figure 1. The first one is the reference with density and Young's Modulus $\left(\rho_{o}, E_{o}\right)$ and performance, $p_{o}$, while the second is a candidate cross-section with properties $(\rho, E)$.

The material index to estimate the performance $p$ of a candidate relative to that of the reference is given by $[6,8]$ :

$$
\frac{p}{p_{o}}=\left(\frac{E}{E_{o}}\right)^{q} \frac{\rho_{o}}{\rho}
$$

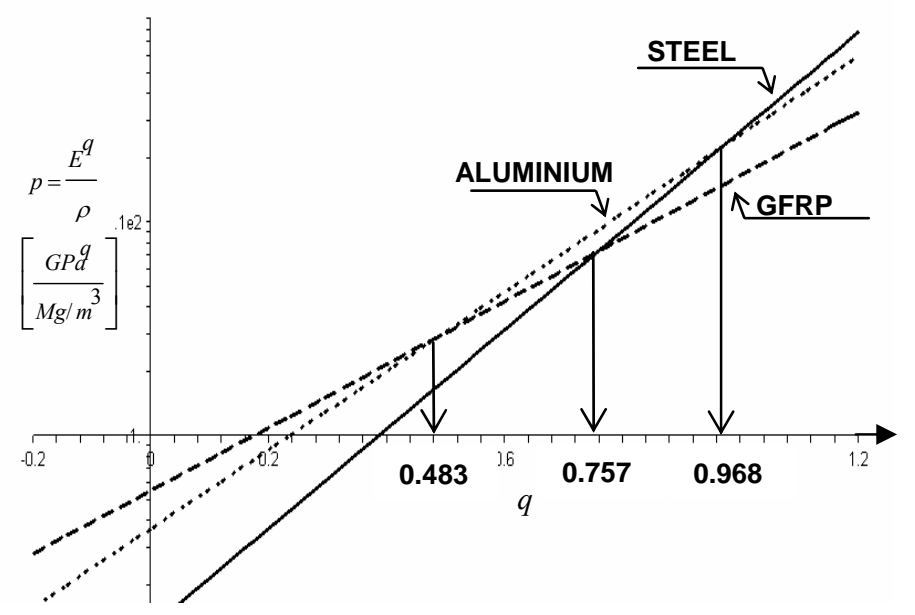

Figure 3. Performance index plotted against the scaling parameter, $q$, for material selection

\begin{tabular}{|c|c|c|}
\hline \multicolumn{1}{|c|}{ Range } & Material Ranking & $\begin{array}{c}\text { Ranking Label } \\
\text { for Figure 7 }\end{array}$ \\
\hline$-\infty<q<0.483$ & $\begin{array}{c}\text { GFRP } \\
\text { ALUMINIUM } \\
\text { STEEL }\end{array}$ & G-A-S \\
\hline $0.483<q<0.757$ & $\begin{array}{c}\text { ALUMINIUM } \\
\text { GFRP } \\
\text { STEEL }\end{array}$ & A-G-S \\
\hline $0.757<q<0.968$ & $\begin{array}{c}\text { ALUMINIUM } \\
\text { STEEL } \\
\text { GFRP }\end{array}$ & A-S-G \\
\hline $0.968<q<+\infty$ & $\begin{array}{c}\text { STEEL } \\
\text { ALUMINIUM } \\
\text { GFRP }\end{array}$ & S-A-G \\
\hline
\end{tabular}

Table 3. Material ranking for given $q$ range where the scaling parameter, $q$, is a function of the relative changes of the width, $u$, and of the depth, $v$, of the cross-sections and is:

$$
q=\frac{\ln u v}{\ln u v^{3}}
$$

When the cross-section scaling is proportional, vertical or horizontal, the expression (7) respectively takes the values $1 / 2,1 / 3,1$.

We now use expression (6) to examine how the performance changes with the scaling parameter. Three candidate materials are considered: Aluminium, Steel, and Glass Fibre Reinforced Plastic (GFRP).

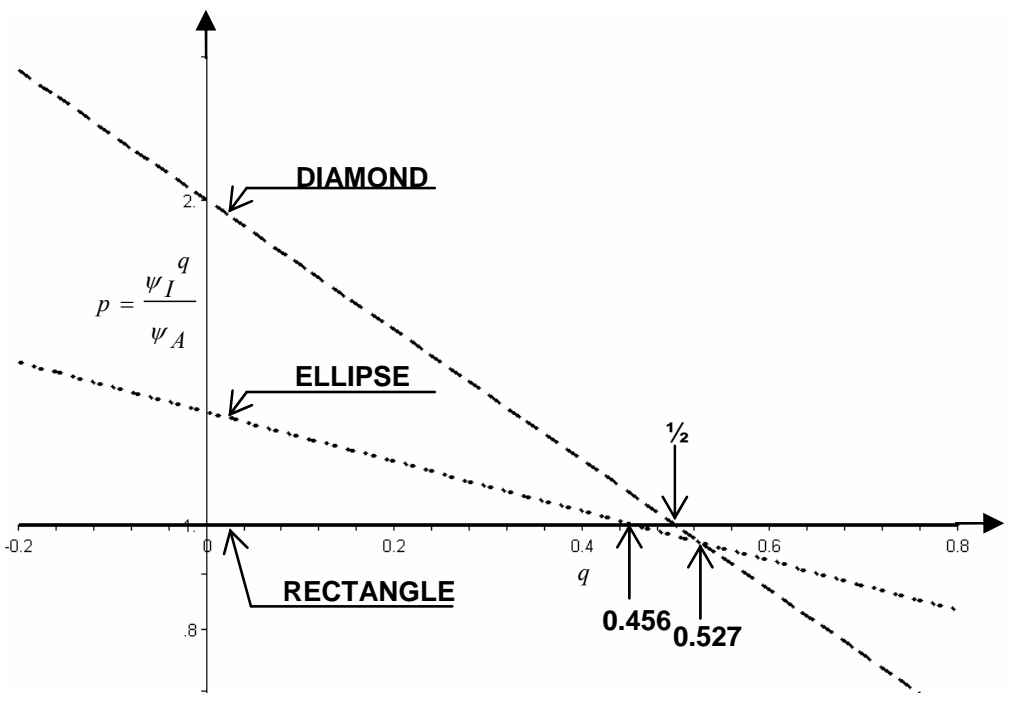

Figure 4. Performance index for shape selection, versus the scaling parameter, $q$.

\begin{tabular}{|c|c|c|}
\hline \multicolumn{1}{|c|}{ Range } & Shape Ranking & $\begin{array}{c}\text { Ranking Label } \\
\text { for Figure 8 }\end{array}$ \\
\hline$-\infty<q<0.456$ & $\begin{array}{c}\text { DIAMOND } \\
\text { ELLIPSE } \\
\text { RECTANGLE }\end{array}$ & D-E-R \\
\hline $0.456<q<1 / 2$ & $\begin{array}{c}\text { DIAMOND } \\
\text { RECTANGLE } \\
\text { ELLIPSE }\end{array}$ & D-R-E \\
\hline $1 / 2<q<0.527$ & $\begin{array}{c}\text { RECTANGLE } \\
\text { DIAMOND } \\
\text { ELLIPSE }\end{array}$ & R-D-E \\
\hline & $\begin{array}{c}\text { RECTANGLE } \\
\text { ELLIPSE }\end{array}$ & R-E-D \\
& $\begin{array}{c}\text { DIAMOND } \\
0.527<q<+\infty\end{array}$ & \\
\hline
\end{tabular}

Table 4. Shape ranking for given $q$ range 
The performance index is plotted as a function of the scaling parameter $q$ in Figure 3 using material properties given in Table 2. The change of the material performance with $q$ shows that the direction of scaling has an impact on the selection of the best material. The plot illustrates that two materials have the same performance index for special values of $q$, which are given by the intersections of their performance curves. For example, 0.483 is the value of $q$ where GFRP and Aluminium perform equally. For $q=0.968$, Steel has the same performance as Aluminium.

The special values of $q$ in Figure 3 can be used to define ranges of scaling where the performance ranking of candidate materials does not change. For example, for a given stiffness, the range $0.483<q<$ 0.757 is characterized by a performance ranking where Aluminium is always the best compared to the others, while Steel is the worst. Other material rankings for the whole range of scaling conditions, $q$, are shown in Table 3.

\subsection{Shape selection}

The general index to select the lightest crosssectional shape of a beam with given bending stiffness, material, and length is [6,7]:

$$
\bar{p}_{s}=\frac{\psi_{I}^{q}}{\psi_{A}}
$$

where $q$ is given by expression (7).

Similarly to the analysis of the material index, we now use equation (8) to examine how scaling affects the performance of Shapes. Three candidate cross-sections are examined: rectangle, ellipse, and diamond. The Shape Transformers (Table 1) have been used to plot expression (8) in Figure 4. The full range of solutions is given for any scaling condition. When Figures 3 and 4 are compared, it can be observed that the Shape Transformers $\left(\psi_{A}, \psi_{I}\right)$ are properties that play a similar role to that of the material properties $(\rho, E)$ in determining performance.

Figure 4 shows that the performance of the reference cross-section does not change for any scaling because the rectangle is also the Envelope of the Shape and has $\psi_{A}=\psi_{I}=1$. Similarly to material selection, the special values of $q$ are the intersection points of two performance curves and describe the relative scaling for which both shapes perform equally. These separate $q$ ranges where the shape ranking of the candidates does not vary. For example, $q$ less than 0.456 is a scaling range where a diamond is always lighter than an ellipse, which is in turn better than a rectangle. Shape rankings for the full ranges of scaling are summarized in Table 4.

\subsection{Material and Shape co-selection}

When Material and Shape attributes are crosssection variables, the general performance index, $\bar{p}$, [6,7] for minimising the mass of a stiff beam with any scaling of the Envelope is:

$$
\bar{p}=\frac{\left(E \psi_{I}\right)^{q}}{\rho \psi_{A}}
$$

where $q$ is given by expression (7).

Table 5 gives a summary of the selection indices based on the Shape Transformers method applied to bending stiffness design. While $q$ for arbitrary scaling depends on the relative changes of the cross-section Envelopes and thus does not have one single value, $q=1, q=1 / 3$, and $q=1 / 2$ are specific of a scaling transformation and are found by inserting the values of the multiplicators in expression (7).

Graphical co-selection of Material and Shape has been recently examined for the three conditions of scaling on charts with axes $\left(\rho \psi_{A}, E \psi_{I}\right)$ [10]. Section 4 will examine graphical selection for arbitrary scaling.

\begin{tabular}{|c|c|c|c|}
\hline Selection & Material & Shape & $\begin{array}{c}\text { Material } \\
\text { and Shape }\end{array}$ \\
\hline $\begin{array}{c}\text { Arbitrary, } \\
u \neq v \neq 1\end{array}$ & $\frac{E^{q}}{\rho}$ & $\frac{\psi_{I}^{q}}{\psi_{A}}$ & $\frac{\left(E \psi_{I}\right)^{q}}{\rho \psi_{A}}$ \\
\hline $\begin{array}{c}\text { Horizontal, } \\
v=1\end{array}$ & $\frac{E}{\rho}$ & $\frac{\psi_{I}}{\psi_{A}}$ & $\frac{E}{\rho} \lambda$ \\
\hline $\begin{array}{c}\text { Vertical } \\
u=1\end{array}$ & $\frac{E^{1 / 3}}{\rho}$ & $\frac{\psi_{I}^{1 / 3}}{\psi_{A}}$ & $\frac{\left(E \psi_{I}\right)^{1 / 3}}{\rho \psi_{A}}$ \\
\hline $\begin{array}{c}\text { Proportional, } \\
u=v\end{array}$ & $\frac{E^{1 / 2}}{\rho}$ & $\frac{\psi_{I}^{1 / 2}}{\psi_{A}}$ & $\frac{\left(E \psi_{I}\right)^{1 / 2}}{\rho \psi_{A}}$ \\
\hline
\end{tabular}

Table 5. Performance indices

\section{Selection charts}

The first and second parts of this section explore the visualization of the selection indices on charts that plot the material and the shape properties on log axes. The third part proposes an alternative approach of selection consisting of limiting regimes of shape and material performance. 


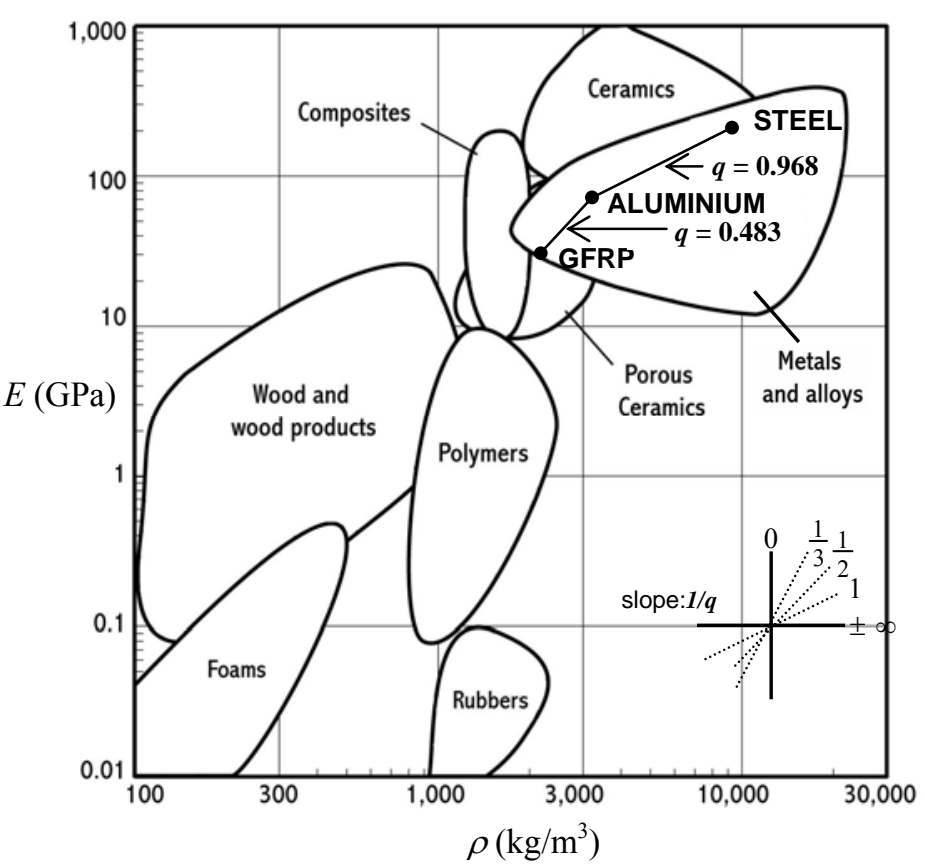

Figure 5a. Adapted Material property charts, $E$ vs. $\rho$. (Chart courtesy of M. F. Ashby [5])

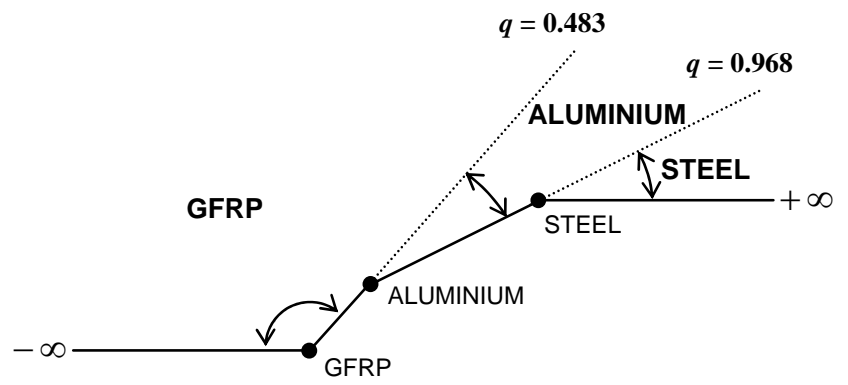

Figure $\mathbf{5 b}$. Zoom of Figure 5 a showing regions bounded by limiting slope of $q$, where a material has a better performance compared to other candidates

\subsection{Material property charts}

Logarithmic scale charts are commonly used in material selection to display the wide range of material properties. Figure $5 \mathrm{a}$ is an example of a chart with $\rho$ vs. $E$ [5]. To plot the general expression of the material index on such a chart, we rearrange and take the log of equation (6) such that:

$$
\log \frac{E}{E_{o}}=\frac{1}{q} \log \frac{\rho}{\rho_{o}}+\frac{1}{q} \log \frac{p}{p_{o}}
$$

Plotted in Figure 5a, Equation (9) represents an iso-performance line with slope $1 / q$ and y-intercept

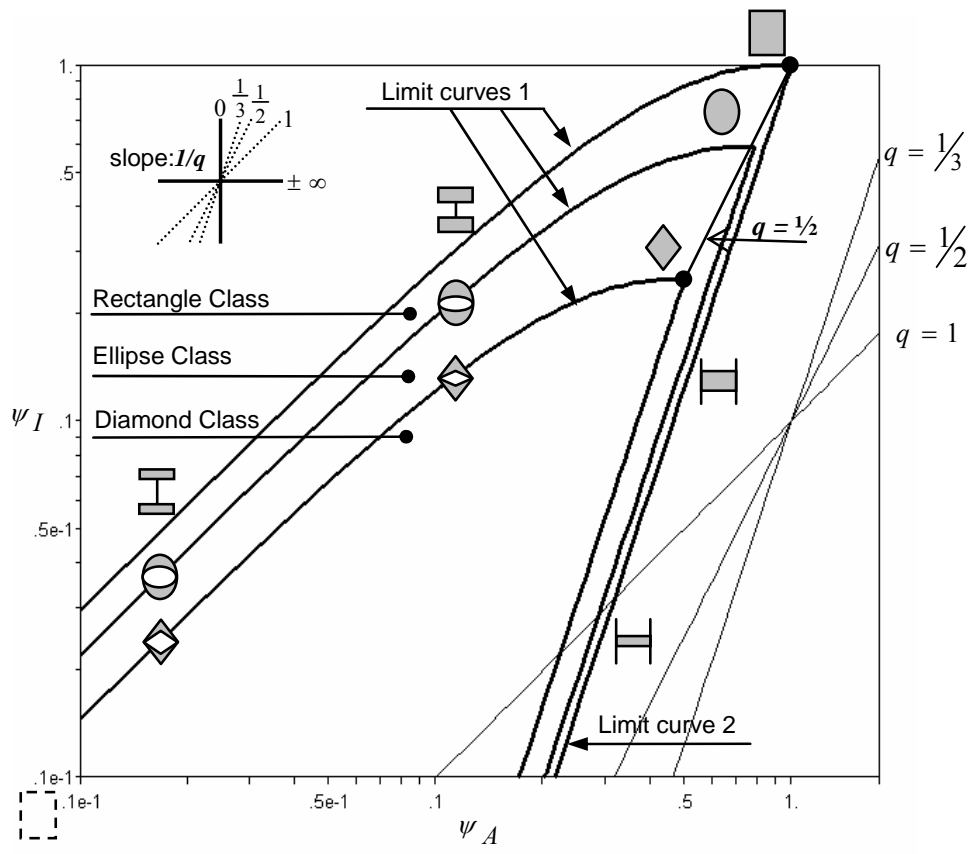

Figure 6 a. Shape property charts, $\psi_{I}$ vs. $\psi_{A}$. (log-log scale)

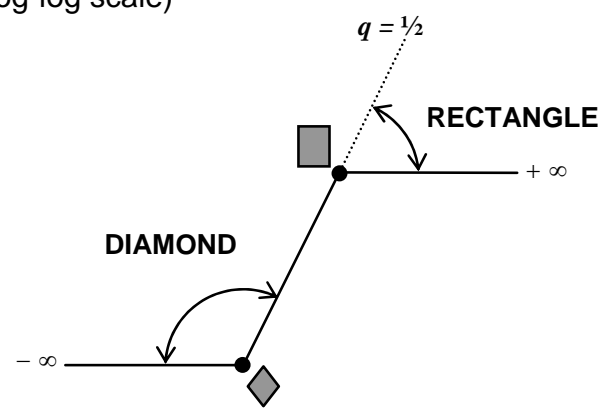

Figure 6b. Zoom of Figure 6a showing regions bounded by limiting slope of $q$, where a shape performs better than the others.

$\frac{1}{q} \log \frac{p}{p_{o}}$. Ashby has illustrated how these lines can guide material selection for three scaling conditions of a cross-section [5]. Their use requires a decision regarding cross-section scaling such that a candidate material $(\rho, E)$ will have the same performance of a reference material $\left(\rho_{o}, E_{o}\right)$ if both belong to a guideline. For a given $q$, better candidates are isolated above, while worse below.

Equation (9) is used to visualise the performance of arbitrarily scaled cross-sections in Figures 5a. We first consider GFRP, Aluminium, and Steel as candidate materials, and we identify their positions by using the material properties given in Table 2 . Then we insert the special values of $q$, i.e. the performance curve intersections of Figure 3, in 
equation (9) and we plot the lines on Figure 5a. As expected, it is found that the lines pass through the coordinates describing two materials. For example, 0.483 (Figure 3) is the value of $q$ that defines a scaling condition for which GFRP performs like Aluminium. While the line connecting Steel and GFRP in Figure 5a has a slope of 0.968 , which is given by its special $q$ in Figure 3 .

Figure $5 \mathrm{~b}$ is an expanded view of Figure $5 \mathrm{a}$. Here the top-ranked material among the candidates is also shown for each $q$ range listed in Table 3. Once $q$ is known, the guideline can be plotted to choose the best material for a prescribed scaling. It is noted that material property charts always require evaluating $q$ for a given arbitrary scaling. To avoid this calculation, alternative maps are addressed in Section 4.3 .

\subsection{Shape property charts}

Shape Transformers $\left(\psi_{A}, \psi_{I}\right)$ have been recently used to produce charts $\psi_{A}$ vs. $\psi_{I}$ for a variety of Shapes. Such charts are Envelope Efficiency maps and have been examined to visualize the performance of cross-sections with the same Envelope [9,10]. A simplified example is illustrated in Figure 6a on a log scale to consider the selection of scaled crosssections.

Three classes of shapes are considered: rectangle, ellipse, and diamond. Each class contains cross-sections derived from the solid shape by removing a material layer of the same shape. The performance of each class is identified by a distinct region, whose boundaries are set by the limiting curves 1 and 2. Using the Shape Transformers found in Table 1, these curves have only been plotted for cross-sections that have material layers placed on the upper and lower surfaces, and that are vertically scaled. Curves for other scaling of the internal layer have been examined $[9,10]$ but omitted here to ensure readability.

Like the analysis in the previous section, we examine the plot of the general shape index. Expression (8) is rearranged to display the performance on log scale so that:

$$
\log \psi_{I}=\frac{1}{q} \log \psi_{A}+\frac{1}{q} \log p
$$

Equation (10) is an iso-performance line that can guide selection in Figure $6 \mathrm{a}$ for a given level of performance and scaling. For example, $q=1$ and $q=1 / 3$ are the slopes of the lines for selecting the best shapes in height and width constrained design, while $q=1 / 2$ governs the shape selection for a proportional scaling.

A similar analysis to that explained in section 4.1 applies here. The slope of a line connecting two couples of shape properties $\left(\psi_{A}, \psi_{I}\right)$ in Figure 6a is the special $q$ in Figure 4.

Top-ranked shapes for the $q$ ranges given in Table 4 are displayed in Figure $6 \mathrm{~b}$, which is a zoom of Figure 6a. It is noted that two regions are shown because there is no relative scaling for a solid ellipse to be lighter than both a rectangle and a diamond (Figure 4).

While $q$ is always known for horizontal, vertical, and proportional scaling, other conditions require $q$ to be estimated before plotting its line on the chart.

\subsection{Limiting regime maps}

Figure 3 and 4 are selection charts in the domains of Material and Shape properties. The selection guidelines are superimposed to represent the performance for a given scaling imposed on the cross-sections. While horizontal, vertical, and proportional scaling are identified by three $q$ values (Table 3), for arbitrary scaling, $q$ changes with the scaling transformation and thus an additional estimation is needed.

To avoid this encumbrance, an alternative is to approach the selection in the domain of the Envelope size. We plot curves of the special values of $q$, i.e. intersections in Figure 3 and 4, on charts $u$ vs. $v$. For a given $q$, the change of $v$ with variation of $u$, can be found by inverting equation (7) so that:

$$
v=u^{\frac{(1-q)}{(3 q-1)}}
$$

which can also be written as:

$$
H_{1}=H_{o}\left(\frac{B_{1}}{B_{o}}\right)^{\frac{(1-q)}{(3 q-1)}}
$$

Equations (11) and (12) can be plotted on charts $u$ vs. $v$ for the special values of $q$ obtained from Figures 3 and 4 . The curves define limiting regimes of performance ranking in the design space of the cross-sections. Limiting regime maps have the advantage of displaying performance rankings in a domain where any geometric constraints can be directly applied. Materials and Shapes can be promptly selected for any scaling condition and with no calculation. This is shown in the following sections. 


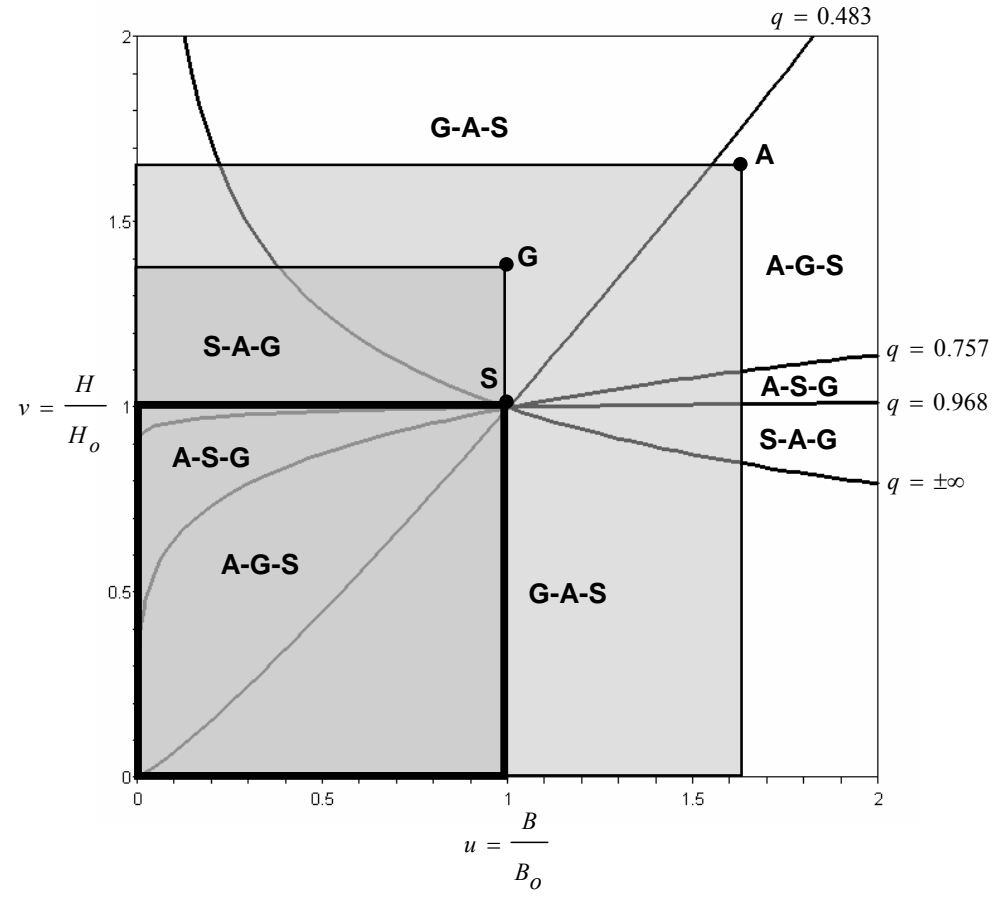

Figure 7. Material limiting regime chart.

\subsubsection{Material limiting regime maps}

Figure 7 shows the material regime map for three competing materials: Steel, Aluminium, and GFRP. Equation (11) is used to plot the change of $v$ with $u$ for the special values of $q$ (Figure 3). The curves of the material regime map delimit regions of the design space where the performance of one material is relatively better than the others. The material ranking labels given in Table 3 are displayed in Figure 7 for all ranges of $q$.

Figure 7 permits exploring how Steel, Aluminium, and GFRP compete in the design space. Consider, for example, two design tasks that require the selection of the lightest material for a beam. In the first scenario, a vertical constraint restricts the scaling of the candidate cross-sections, such as $\mathrm{S}$ and $\mathrm{G}$ in Figure 1. While in the second one, the designer chooses to consider cross-sections that are proportionally scaled, such as S and A in Figure 1.

The map of Figure 7 gives prompt answer to the problems. GFRP $(G)$ is better than Steel for vertical scaling since $\mathrm{G}$ lies in the region where the ranking is G-A-S $(q<0.483)$. In the second case, Aluminium is lighter than Steel because the scaling transformation applied to the cross-sections is such that A lies in the A-G-S ranking regime.

The material regime map can be developed for a group of pre-selected materials available to a designer, and then used to select the best candidate for a cross-section subjected to any scaling condition.

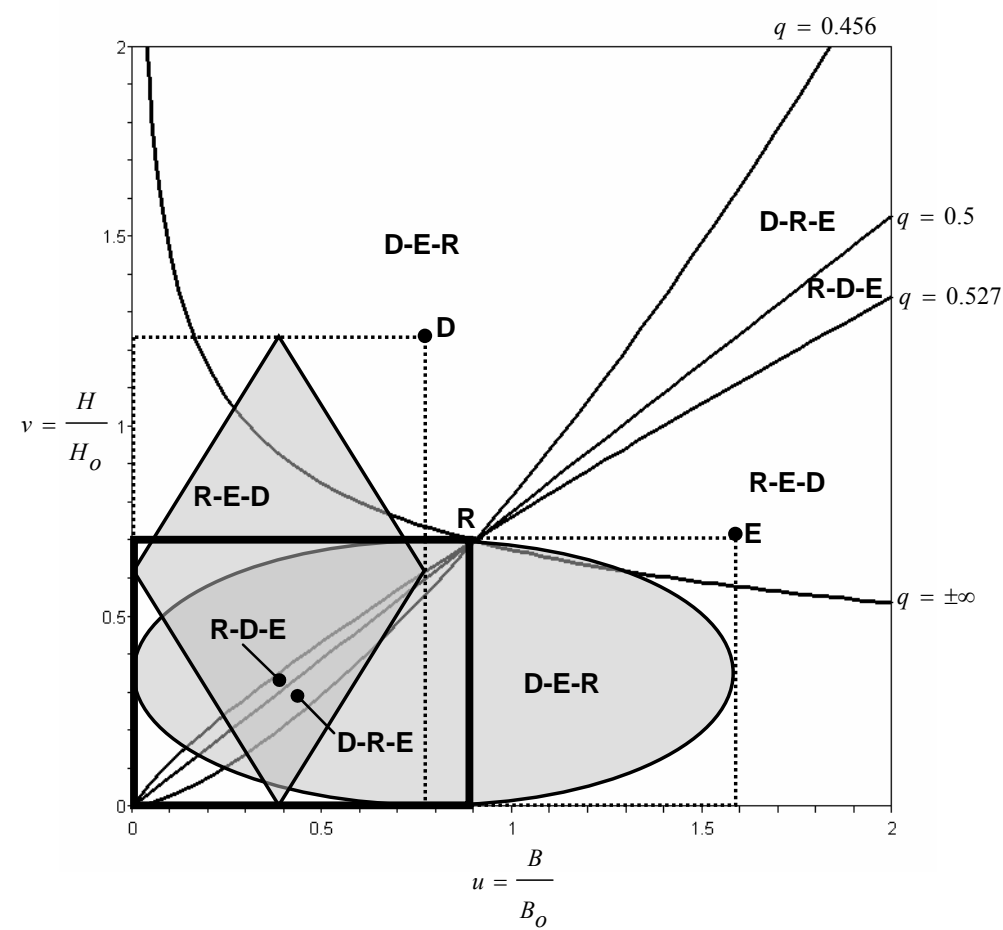

Figure 8. Shape limiting regime chart.

\subsubsection{Shape limiting regime maps}

Similarly to Figure 7, a limiting regime map for three competing shapes is illustrated in Figure 8. The special values of $q$ (Figure 4) are inserted in equation (12) to plot the limiting curves for rectangle, ellipse, and diamond. This time the reference cross-section is not a square but a generic rectangle, $\mathrm{R}$. The labels representing a shape ranking (Table 4) are displayed for given ranges of $q$. The ranking indicates how shapes performance changes with scaling.

For example, Figure 8 can be used to compare the performance of the rectangle to that of the diamond, D, and ellipse, E, in Figure 1b. The map shows that the arbitrary scaling of the cross-section $\mathrm{D}$ relative to the reference, $R$, is such that $D$ lies in the D-E-R ranking region and thus the rectangle will result in being heavier. Different results are obtained if a height constraint imposes a horizontal scaling to the cross-sections, such as $\mathrm{R}$ and $\mathrm{E}$. The ellipse, E, is in the region R-E-D, where the rectangle has a better performance.

The limiting regime map can give insight into the selection of the best shape among a number of pre-selected shapes.

\section{Concluding remarks}

This paper has presented a graphical exploration of the performance indices for material and shape 
selection. The general solutions of the indices have been obtained by using the method of the Shape Transformers, which are dimensionless measures of the area $\left(\psi_{A}\right)$ and second moment of area $\left(\psi_{I}\right)$ of a cross-section and thus are invariant, alike materials, to macroscopic scaling.

When plotted on material and shape property charts ( $\rho$ vs. $E$ and $\psi_{A}$ vs. $\left.\psi_{I}\right)$, the general expression of the selection indices can be used as isoperformance lines that guide selection for any scaling imposed on a cross-section. It has been shown that while property charts are handy for selecting crosssections scaled either horizontally, vertically, or proportionally, they may result in being not so versatile in dealing with arbitrary scaling.

This paper has addressed the issue and has proposed limiting regime maps. These charts display on their axes the relative change of the cross-section sizes ( $u$ vs. $v)$, and illustrate how shapes and materials compete in the design space. For specific values of the scaling parameter $q$, limiting curves are plotted to define performance-ranking regimes for materials and shapes. The maps give insight into how scaling impacts their selection.

\section{Acknowledgement}

The author wishes to thank Professor M.F. Ashby of Cambridge University (U.K.) for authorizing the use of the chart $E$ vs. $\rho$, and Ms. Stephanie Rinaldi, undergraduate of Mechanical Engineering at McGill University, for the assistance in editing the illustrations.

\section{References}

[1] Cox, H.L., 1965, The design of structures of least weight, Pergamon Press, Oxford.

[2] Shanley, F. R., 1960, Weight-strength Analysis of Aircraft Structures, $2^{\text {nd }}$ edition, New York, Dover

[3] Caldwell, J.B. and Woodhead R.G., 1973, "Ship structures: some possibilities for improvement", North East Cost Institution - Institution Engineers \& shipbuilders - Transaction, 89, pp. $101-120$

[4] Ashby, M. F., 1991, "Materials and shape". Acta Metall. Mater.,. 39(6), pp.1025 - 1039.

[5] Ashby, M. F., 1999, Material selection in mechanical design, $2^{\text {nd }}$ edition, Pergamon Press, Oxford.

[6] Pasini, D., 2003, "A new theory for modelling the mass-efficiency of material, shape and form", $\mathrm{PhD}$ thesis, Bristol University.

[7] Pasini, D., Smith D.J, Burgess, S. C., 2003, "Selection of arbitrarily scaled cross-sections in bending stiffness design” Proc. Instn Mech. Engrs,
Part L, Journal of Materials: Design and Applications, 217 (2), pp. 113-125

[8] Pasini, D., Burgess, S. C., Smith D.J, 2002, "Performance indices for arbitrarily scaled rectangular cross-sections in bending stiffness design", Proc. Instn Mech. Engrs, Part L, Journal of Materials: Design and Applications, 216, pp. 101-113

[9] Pasini, D., Smith D.J, Burgess, S. C., 2003, "Structural efficiency maps for beams subjected to bending", Proc. Instn Mech. Engrs, Part L, Journal of Materials: Design and Applications, 217 (3), pp. $207-$ 220.

[10] Pasini, D., 2005, "Shape Transformers for Material and Shape Selection", Proceedings of IDETC/DTM 2005, ASME 2005 International Design Engineering Technical Conference \& Computers and Information in Engineering Conference, Long Beach, California, USA. 